\title{
Morbus Gaucher
}

\section{Enzymersatz mit Velaglucerase alfa - Seit 5 Jahren bewährte therapeutische Strategie}

Morbus Gaucher ist eine autosomal rezessiv vererbte, lysosomale Speicherkrankheit. Die unspezifischen Symptome erschweren es dem Arzt, die richtige Diagnose zu stellen. Durchschnittlich vergehen 9 Jahre bis zum Therapiebeginn dieser seltenen Erkrankung. Dabei stehen für die Behandlung gute Optionen zur Verfügung, z.B. die Enzymersatztherapie Velaglucerase alfa $\left(V P R I V^{\circledR}\right)$ [1]. Langzeitdaten der seit 5 Jahren in Deutschland erhältlichen Therapie bestätigen die gute und nachhaltige Wirkung sowie die gute Verträglichkeit der Substanz. Bei einem Presse-Roundtable der Shire Deutschland GmbH sprachen Experten über ihre Erfahrungen mit der Erkrankung und den Therapiemöglichkeiten.

Bei Patienten mit Morbus Gaucher besteht ein Mangel des Enzyms Glucocerebrosidase, das in den Zellen überschüssiges Glucocerebrosid in Glucose und Ceramid spaltet. Aufgrund des Glucocerebrosidase-Mangels speichern die Lysosomen zu viel der fetthaltigen Substanz Glucocerebrosid - es kommt zu einer Vergrößerung der Makrophagen, die dann als Gaucher-Zellen bezeichnet werden. Die Funktionsbeeinträchtigung kann verschiedene Organe betreffen. Häufig besteht eine starke Vergrößerung von Milz und Leber und eine Abnahme der Knochendichte. Bei einer Erstmanifestation des Morbus Gaucher im Kindesalter können eine Blutungsneigung, Knochenschmerzen, ein vorgewölbtes Abdomen und Entwicklungsverzögerungen auf die Erkrankung hinweisen.

\section{Entscheidend: Rechtzeitige Behand- lung durch frühe Diagnose}

Prof. Dr. Stephan vom Dahl, Klinik für Gastroenterologie, Hepatologie und Infektiologie am Universitätsklinikum Düsseldorf, erinnerte an die wichtige Rolle der Diagnose. Ein unbehandelter Morbus Gaucher resultiere in einer Minderung der Lebensqualität, Zerstörung des Knochens, Invalidität sowie einer verkürzten Lebenserwartung. Da einfache und sehr wirksame Therapiemöglichkeiten bestehen, seien die Früherkennung und kompetente Einleitung der Therapie besonders wichtig, konstatierte vom Dahl. Eine Diagnose kann mittels eines Trockenbluttests und/ oder einer einfachen Blutentnahme erfolgen. Für die Therapie des Morbus Gaucher stehen Substanzen zweier Wirkmechanismen zur Verfügung - die kausale Enzymersatztherapie sowie die Substratreduktionstherapie, die den Aufbau der Speichersubstanz reduziert. Velaglucerase alfa ist die einzige auf humanen Zelllinien basierende Enzymersatztherapie.

\section{Auf einen Blick - Typische Krankheitszeichen und Symptome des Morbus Gaucher Typ 1*}

Hämatologisch

Anämie, Thrombozytopenie, Leukopenie

Erhöhte Inzidenz eines Multiplen Myeloms MGUS

Viszeral

Splenomegalie, Hepatomegalie, interstitielle Lungenerkrankung

Ossär

Knochen- und Gelenkschmerzen, Knocheninfarkte/Knochennekrosen, Osteopenie/Osteoporose, pathologische Frakturen, Osteosklerose, Knochenkrisen, Erlenmeyer-Deformationen
ZNS/PNS

Parkinsonismus, Polyneuropathie, periphere Neuropathie

Metabolisch und Sonstige

Fatigue, erhöhter Grundumsatz, Untergewicht, Wachstumsverzögerung, Entwicklungsverzögerung

Bei Verdacht auf Morbus Gaucher bietet die Shire Deutschland GmbH einen kostenlosen Diagnoseservice an:

Tel.: 030206 582-0; Fax: 030206 582-100

Email: diagnostikservice@shire.com

"Morbus-Gaucher-Patienten können mehrere oder auch nur eines der aufgeführten Symtome aufweisen.

\section{Enzymersatztherapie auch für} Kinder und Schwangere zugelassen Die orale Verfügbarkeit, als Vorteil der Substratreduktionstherapie gegenüber der Enzymersatztherapie, werde durch die Anwendungsbeschränkungen, die vielen möglichen Wechselwirkungen mit Nahrungsmitteln und anderen Arzneimitteln sowie der zu erwartenden Non-Compliance begrenzt, bemerkte Prof. Dr. Georg Kojda, Institut für Pharmakologie und Klinische Pharmakologie, Heinrich-Heine-Universität Düsseldorf. Für die Enzymersatztherapie seien dagegen keine Wechselwirkungen mit anderen Arzneistoffen bekannt, so Kojda. Ein weiterer Vorteil sei, dass die Enzymersatztherapie auch bei Kindern, älteren Patienten oder in der Schwangerschaft eingesetzt werden könne.

\section{Langzeitwirksamkeit von Velaglucerase alfa}

Dr. Eugen Mengel, Villa Metabolica, Johannes-Gutenberg-Universität Mainz, stellte Daten zur Wirksamkeit von Velaglucerase alfa vor, wonach 57 Patienten der Zulassungsstudien bis $\mathrm{zu} 4,8$ Jahre mit $60 \mathrm{U} / \mathrm{kg} \mathrm{VPRIV}^{\circledR}{ }^{\circledR}$ weiterbehandelt wurden. Die signifikanten Therapieerfolge der ersten 2 Jahre hinsichtlich hämatologischer Endpunkte wie der Verbesserung der Hämoglobinkonzentration (26\%), Erhöhung der Blutplättchen (120\%) und ebenso die Reduktion der Organvolumina der Leber (27\%) und der Milz (64\%) wie auch der Biomarker blieben im weiteren Behandlungsverlauf erhalten [2]. Eine weitere Studie über 7 Jahre bestätigt die Ergebnisse dieser Extensionsstudie [3].

Die Infusionen mit VPRIV ${ }^{\circledR}$ sind gut verträglich und können bei Patienten, die mindestens 3 Ap- plikationen erhalten haben, unter Aufsicht einer medizinischen Fachkraft auch zuhause verabreicht werden.

\section{Fazit}

Das Auftreten von irreversiblen Organschäden und Skelettkomplikationen bei Morbus Gaucher kann mit der bewährten Enzymersatztherapie Velaglucerase alfa verhindert werden, wenn die Behandlung dank einer frühen Diagnose rechtzeitig begonnen wird. In zwei aktuellen Auswertungen von Langzeitbeobachtungen wurden die Wirksamkeit und Sicherheit der Enzymersatztherapie mit Velaglucerase alfa bestätigt.

Dr. Ine Schmale, Westerburg

\section{Quelle}

Presse-Roundtable der Shire Deutschland $\mathrm{GmbH}$ «Bewährte Enzymersatztherapie bei Morbus Gaucher - 5 Jahre VPRIV $^{\circledR}$ ", 5. Juni 2015, Frankfurt/M.

\section{Literatur}

1 Fachinformation VPRIV ${ }^{\circledR}$, Stand Dezember 2013.

2 Hughes DA et al.: Am J Hematol 2015; 90:584-591.

3 Zimran A et al.: Am J Hematol 2015; 90:577-583.

\section{Impressum}

\section{Morbus Gaucher}

Enzymersatz mit Velaglucerase alfa -

Seit 5 Jahren bewährte therapeutische

Strategie

PharmaForum in

OnCology Research AND TREATMENT 38 | 9 | 15

(c) 2015 by S. Karger Verlag für Medizin

und Naturwissenschaften $\mathrm{GmbH}$

Wilhelmstraße 20A

79098 Freiburg, Deutschland

Mit freundlicher Unterstützung durch

Shire Deutschland GmbH

Verlag, Herausgeber, Redaktion und Verlagsgeschäftsführun übernehmen keine Verantwortung für den Inhalt dieser Rut 